\title{
A Reliability Evaluation for Distribution Network Under V2G Mode Considering Random Behavior Of Electric Vehicles Charge and Discharge
}

\author{
Xiaoquan $\mathrm{Li}^{1, \text { a }}$, Shuai Liü, b Jianfeng $\mathrm{Liu}^{2, \mathrm{c}}$ and Xiaoliang You ${ }^{1, \mathrm{~d}}$ \\ ${ }^{1}$ College of Air and Missile Defense, Air Force Engineering, Shaanxi 710051, China; \\ ${ }^{2}$ Zhengzhou Jinyang Electric Co., Ltd, Henan 450006, China. \\ alxq389@163.com, bmasteryliu@163.com, ${ }^{\mathrm{c}} \mathrm{ljf} @ 163 . c o m,{ }^{\mathrm{d}}$ you0961@163.com
}

Keywords: Distribution network, Reliability evaluation, Electric vehicles; V2G.

\begin{abstract}
Reliability evaluation of the electric vehicle in the grid of random charging and discharging behavior is particularly important. To solve these problems, a mode is proposed in the time and space by their random behavior, probability density function based on the statistical analysis, and the bind distribution model based on the blind number theory were obtained respectively. Meanwhile, the reliability of RBTS-BUS6 is evaluated by an improved Monte Carlo simulation method based on dual sampling, The results shown that electric vehicles random behavior under a variety of factors quantitative have a significant impact on the level of grid reliability standards, provided a useful basis for the future of its orderly play an active role in the distribution network.
\end{abstract}

\section{Introduction}

At present domestic and international research in this area is still relatively small. Literature [1] has shown that reliability modeling and quantitative analysis of distribution network considering different types of electric vehicles, mainly analyzed on the fixed access point and permeability under V2G mode. Literature [2] mainly proved a massive PHEV connected to the generation and transmission system would be severely affected the reliability of the grid, which is focusing on the establishment of a reliability evaluation model and measurements in detail. The above-mentioned literature has been restricted the random acts of electric vehicles charge and discharge, it makes the reliability assessment results are biased in favor of optimistic scenario.

The randomness behavior of electric vehicles charge and discharge is mainly reflected in time and space. The time is mainly connected to the charge from the start to end time, and the space is mainly connected to the charge loading size and the access location. The electricity price adjustment mechanism and effective control strategy is used to constrain time in the literature [1,2], but both cases in the existing power system are difficult to achieve.

Based on the statistical data of electric vehicles travel characteristic, molded the probability density function from the perspective of time and space for various factors which affect the electric vehicle random behavior. And then sampled the probability density function which is established above, analyzed the impact of the electric vehicle random behavior on the distribution network reliability levels by an example.

\section{The load model of electric vehicle random charge}

\subsection{Load model of charge time}

Randomness of electric vehicles charge time led the effect directly from the "peaks plus Peak" or "load shifting" in the grid. The probability model established by electric vehicles charge time in this paper is based on the analysis of private passenger data of Beijing[3] and the survey result of the Nation's family of vehicles[4]. Because of the current is not constant problem on the three-stage charge method of electric vehicles, the paper is assumed that the current in charge process is constant, and the error after assumption among with the error caused by different demand of charge power due 
to different remaining capacity are included in the standard of charge time. Not only simplifies the value which is estimated by different factors distribution models, but also includes the effect on the grid in the reliability assessment process, which is greatly improved the accuracy of reliability evaluation.

The statistical result showed that the return and travel time of electric vehicles is subject to the normal distribution according to the literature [3] [4]. The time to end and start the charge is assumed the electric vehicle return and travel time, then the time to start and end charge $T_{s}, T_{e}$ of the electric vehicle is also subject to the normal distribution, the probability density function is:

$$
f_{T_{s}}=\frac{1}{\sigma_{T_{s}} \sqrt{2 \pi}} \exp \left[-\frac{\left(x-\mu_{T_{s}}\right)^{2}}{2 \sigma_{T_{s}}^{2}}\right], f_{T_{e}}=\frac{1}{\sigma_{T_{e}} \sqrt{2 \pi}} \exp \left[-\frac{\left(x-\mu_{T_{e}}\right)^{2}}{2 \sigma_{T_{e}}^{2}}\right]
$$

Where: $\mu_{T_{s}}, \sigma_{T_{s}}, \mu_{T_{e}}, \sigma_{T_{e}}$ respectively, the start and end time of the charge means and standard deviations. Different power charge requirement will be included in the error of charge time, in the $\sigma_{T_{s}}, \sigma_{T_{e}}$. The distribution in time of electric vehicle charge load random behavior is sampled to the distribution function in time, the following is described charge load in space.

\begin{tabular}{ccccc}
\multirow{2}{*}{ Table 1 Probability distribution parameters about EV charge time } \\
\cline { 2 - 5 } time & \multicolumn{2}{c}{ Time to start charge } & \multicolumn{2}{c}{ Time to stop charge } \\
\cline { 2 - 5 } & $\mu_{T_{s}} / h$ & $\sigma_{T_{s}} / h$ & $\mu_{T_{e}} / h$ & $\sigma_{T_{e}} / h$ \\
workday & 18 & 3 & 7 & 3 \\
weekend & 15 & 6 & 9 & 6
\end{tabular}

\subsection{Space model of charge load based on Blind}

The object of this paper focuses on the actual process of random characteristics, according to the actual situation of randomly generated the size of electric car, and access points and the size of electric vehicles is not limited by man-made, both of the affect having the characteristics of uncertain information, and therefore the paper introduce a concept of "blind information "[5]. It is not divided uncertain relationship between the two affect while the random nature of the information is described. It makes the model more in line with the process of sampling the actual process of random characteristics.

By analyzing the sales data of the existing electric vehicles and predicting the future conditions, the load of the device may appear in $m$ intervals, $g_{1}=\left[a_{1}, b_{1}\right], g_{2}=\left[a_{2}, b_{2}\right], \cdots, g_{m}=\left[a_{m}, b_{m}\right]$, $g_{i}=g(I)(i=1,2, \cdots, m)$. Then the size of electric vehicles will be appeared in the interval of $g_{i}$, and the credibility of it is $a_{i}\left(a_{i} \in[0,1]\right)$, and $\sum_{i=1}^{m} a_{i}=a \leq 1$, thus electric vehicle size of access point about the Blind model can be obtained:

$$
N(x)= \begin{cases}a_{i} & x=g_{i}(i=1,2,3, \cdots, m) \\ 0 & \text { thers }\end{cases}
$$

While the value of $\beta_{i}$ is corresponded to the confidence value of $\alpha_{i}$, so the each point access size of electric vehicles is determined by the value. The electric vehicles access point and size in the grid can be determined by this method.

\subsection{The demand of electric vehicle charge power}

According to the above the electric vehicles probability distribution in time and blind model in space are established, each point demand of electric vehicles charge power model should be

$$
P_{c i}=T_{i} \square N_{i} \quad(i=1,2, \cdots, n)
$$

Which, $P_{c i}$ is the charge power of node $i, A_{i}$ is the charge time subject to probability distributions of time, $N_{i}$ is size of charge subject to the distribution of Blind. 


\section{The power model of electric vehicle random discharge}

Discharge behavior of electric vehicles is also affected by many factors, the main factors which accounted for the remaining battery capacity QC, the size of the discharge period and discharge electric vehicle access points and size. The state of charge SOC value of electric vehicles discharge is not limited and differentiate discussed, but the difference is involved in the discharge time, the battery status and discharge time is separately discussed, fully reflects its discharge randomness while maintaining the status of each electric vehicle. The discharge process is modeled by the three factors.

\subsection{The model of remaining battery capacity}

Some of the parameters affecting is reflected to the user's characteristics of travel after the electric vehicles connected to the grid, the remaining capacity of the electric vehicle's battery QC and user's daily mileage $L$ are closely related. According to statistics [3,4], the electric vehicle's daily mileage is subjected to the lognormal distribution which parameters are ${ }^{\mu_{m}}$ and $\sigma_{m}$, the probability density function is:

$$
f_{L}(x)=\frac{1}{x \sigma_{m} \sqrt{2 \pi}} \exp \left[-\frac{\left(\ln x-\mu_{m}\right)^{2}}{2 \sigma_{m}^{2}}\right]
$$

Among them, the value of $\mu_{m}$ and $\sigma_{m}$ are 3.20 and 0.88 , then the mean and standard deviation of the electric vehicle's daily mileage are:

$$
\mu_{L}=e^{\mu_{m}+\frac{1}{2} \sigma_{m}^{2}} \quad \sigma_{L}=\mu_{L} \sqrt{e^{\sigma_{m}^{2}}-1}
$$

According to the battery consumed capacity $\mathrm{Q}$ is positively associated with daily mileage, the $\mathrm{Q}$ is as follow. Meanwhile, it is assumed that the battery capacity have no other loss except the drive. Electric vehicles battery remaining capacity QC is satisfied to:

$$
\begin{gathered}
Q=L \square E \\
Q C=C-Q
\end{gathered}
$$

Where, $Q$ is the battery consumption, $L$ is the electric vehicle's daily mileage, $E$ is the electric vehicle energy consumption per kilometer, $\mathrm{C}$ is the total capacity of the battery.

By the formula (4) (5) (6) can be derived the probability distribution of electric vehicle battery remaining capacity $Q C$.

Considered in this paper a number of different types of electric vehicles are connected to the grid, because of literature statistic the value is $45 \mathrm{KWh}$.

\subsection{Time model of EV discharge}

The start time of the discharge time is the return time of electric vehicles and the end times is the travel time. Since there is no literature on the electric vehicles parked time statistical data analysis, probability density function cannot be described exactly. Consider the behavior of electric vehicles during the internal work is randomness, a uniform distribution of the electric car parked time is used to estimate its distribution. The approximate probability distribution function is:

$$
f_{f}(x)=\left\{\begin{array}{lr}
\frac{1}{\sigma_{f} \sqrt{2 \pi}} \exp \left[-\frac{\left(x-\mu_{f}\right)^{2}}{2 \sigma_{f}^{2}}\right] & 0 \leq x<\left(\mu_{f}+9\right) \\
\frac{1}{4} \frac{1}{\sigma_{f} \sqrt{2 \pi}} & \left(\mu_{f}+9\right) \leq x \leq\left(\mu_{f}+15\right) \\
\frac{1}{\sigma_{f} \sqrt{2 \pi}} \exp \left[-\frac{\left(x+24-\mu_{f}\right)^{2}}{2 \sigma_{f}^{2}}\right]\left(\mu_{f}+15\right)<x \leq 24
\end{array}\right.
$$

among them $\mu_{f}=0 ; \sigma_{f}=3.4$.

Sampled the probability density function according to the above description, the electric vehicles start moment of discharge probability density distribution is obtained, combined with the results of the sampling probability distribution of the remaining capacity of the battery, the electric vehicle distribution model of discharge time can be obtained.

\subsection{Space model of EV discharge}

Discharge of the spatial distribution of electric vehicles is also modeled by the blind method. The blind model is established by the method have described above, the electric vehicles discharge scale 
interval ${ }^{g_{i}}$ appropriate to take a small combined with the actual situation, the spatial distribution model of EV discharge can be got.

\subsection{Recovery strategy of electric vehicle supply}

When the distribution network fails, the electric vehicles under V2G capabilities control can be equivalent to a constant output current of distributed power. According to the difference of remaining battery capacity QC, the difference of maximum supply range and powered durations are determined by the maximum output power supply $P_{m}$. Suppose supply region: $S_{1}, S_{2}, \cdots S_{i}$, the electric vehicle power supply to the region should be met:

$$
\sum_{j=1}^{n} P_{E V_{j}} \geq \sum_{i=1}^{k} P_{L_{i}}
$$

In which: $\sum_{j=1}^{n} P_{E V_{j}}$ is power supply capacity within the region $S_{i}$ for all electric vehicles; $\sum_{i=1}^{k} P_{L_{i}}$ is the region $S_{i}$ of all loads.

Limited to the current level of development of science and technology, the electric vehicles battery capacity $\mathrm{C}$ is too small, the electric vehicles is not possible for all loss of load distribution network within the target for effective and reliability, therefore, there is adopted an important load power strategy. The important load power strategy is a priority as a class load supply targets, meet the basically needs of high-grade power supply, after the low-level.

$$
\sum_{i=1}^{k} P_{L_{i}}-\Delta P=\sum_{j=1}^{n} P_{E V_{j}}
$$

Where: $\Delta P$ is the reduced load within the power supply area $S_{i}$. The load is reduced from low to high, until the above formula is met. 


\section{Distribution network reliability evaluation under large-scale electric vehicle access}

\subsection{Reliability evaluation algorithm process}

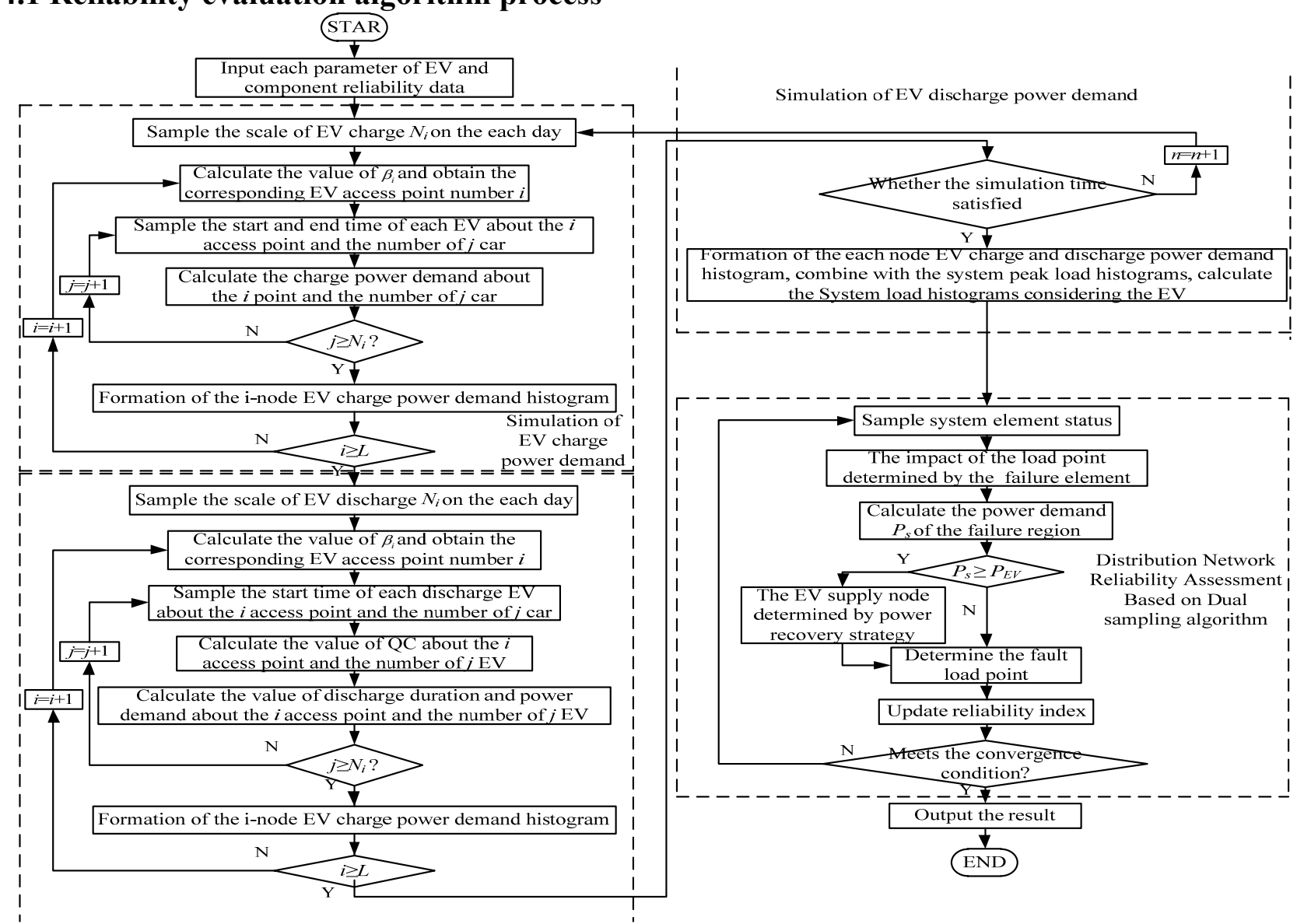

\subsection{Cases Operators}

The RBTS-BUS6-F4 system is given as a case in the article. The structure of the case is shown as above picture, each line is provided an isolation switch. Electric vehicles ownership is assumed that the value for each user is 1.5 , the voltage and current of charge and discharge are $120 \mathrm{~V}$ and $15 \mathrm{~A}$. Other parameters and function of electric vehicles is given by the above, System parameters of each element of reliability parameters and various load points are seen in the reference [6]

The system loads histograms before and after the EV access can be intercepted according to the given parameters and algorithm of the evaluation process by Figure 1.

As can be seen from the figure 1, in case of electric vehicles discharge is not be accounted, small distribution network for each load point load increased significantly, and its impact on the distribution network is more obvious. Under the V2G term of discharge behavior of electric vehicles, the peak load of the system is offset at some extent and the electric vehicle plays a positive role of the system as "load shifting". The impact of electric vehicles random acts on the grid were indicative calculations as follow.

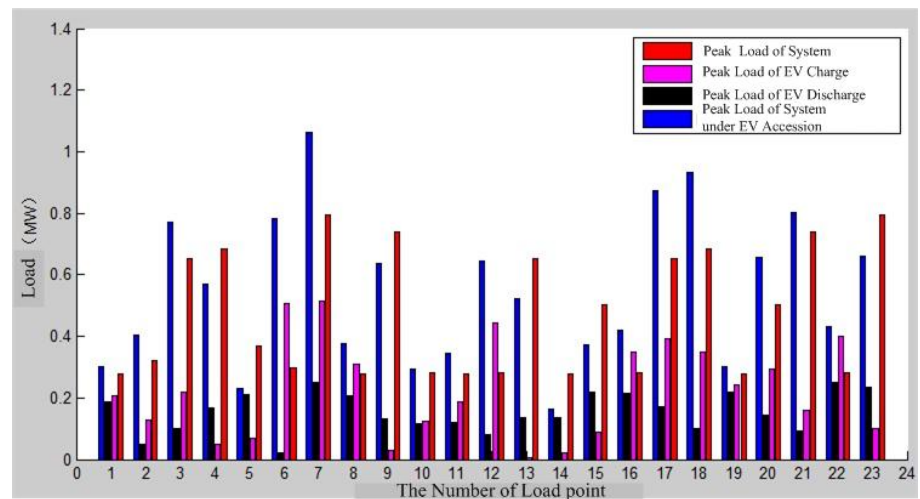

Figure 1 Load histograms of the system 
Three cases of the random behavior are considered in the electric vehicle reliability evaluation, the load level of the three cases as shown:

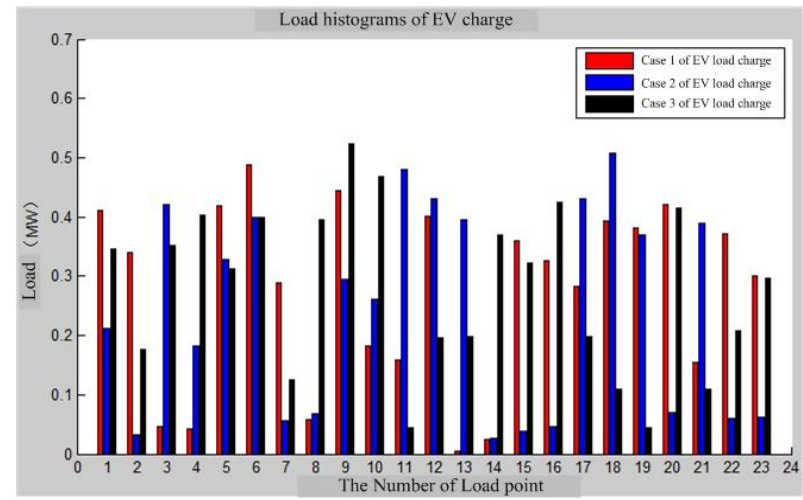

Figure 2 Load histograms of EV charge

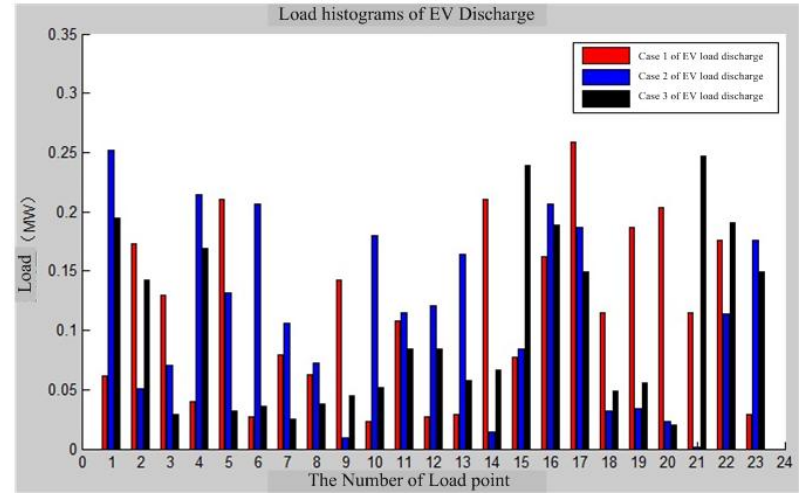

Figure 3Load histograms of EV discharge

As it can be seen from the above three cases load histograms, since the EV each point load level of charge and discharge offset under V2G mode in Case1, making the system Case1 more stable overall load levels; due to each point of the electric vehicle discharge load level is more significant in Case2, and consistent with the level of individual load point more evenly, making Case 2 overall system reliability level than the original system; Case3 since a peak charge load level is created in the load point LP8, LP9, LP10, making the overall system reliability level is lower than the other case. Here are partial load point and system reliability indices.

From Table 1, Table 2, Table 3 reliability indices of partial load point can be drawn: significantly different levels about each point of electric vehicle charge and discharge load is due to the randomness behavior sampling. Reliability levels of load point is fluctuate significantly, in which the biggest indicators of disorderly fluctuations are due to the large gap of the load level caused by EV charge and discharge, a significant impact on the load level of each load point is caused by disorderly, unplanned discharge behavior.

System reliability index can be seen from Table 4, there are significant differences in the reliability level of the system in three different situations, contrast to reliability indices of ordinary distribution network which is given in literature [6] it can be drawn EV randomly charge and discharge behavior have a significant impact on the reliability levels of the distribution network under V2G mode. 
Table 1 Partial load point failure rate

\begin{tabular}{|c|c|c|c|c|c|}
\hline \multirow{2}{*}{ Case } & \multicolumn{5}{|c|}{ Failure Rate $\lambda$} \\
\hline & $L P 1$ & $L P 8$ & LP11 & $L P 16$ & $L P 19$ \\
\hline 1 & 1.6382 & 1.5835 & 1.8925 & 1.9684 & 2.1512 \\
\hline 2 & 1.4850 & 1.5735 & 2.1523 & 1.7216 & 2.4311 \\
\hline 3 & 1.5523 & 1.7352 & 1.7562 & 1.9513 & 2.0212 \\
\hline \multicolumn{6}{|c|}{ Table 3 average recovery time of partial load point } \\
\hline \multirow{2}{*}{ Case } & \multicolumn{5}{|c|}{ Average Recovery Time $r$} \\
\hline & $L P 1$ & $L P 8$ & $L P 11$ & $L P 16$ & $L P 19$ \\
\hline 1 & 4.2646 & 5.2651 & 4.3661 & 5.4651 & 5.6841 \\
\hline 2 & 3.5861 & 5.0553 & 4.5921 & 4.9641 & 5.4954 \\
\hline 3 & 3.9832 & 6.5924 & 3.7562 & 5.2165 & 5.1355 \\
\hline
\end{tabular}

Table 2 Annual average outage time of partial load point

\begin{tabular}{cccccc}
\hline \multirow{2}{*}{ Case } & \multicolumn{5}{c}{ Annual Average Outage Time $u$} \\
\cline { 2 - 6 } & $L P 1$ & $L P 8$ & $L P 11$ & $L P 16$ & $L P 19$ \\
\hline 1 & 7.6160 & 7.7561 & 9.5214 & 9.8135 & 11.2584 \\
\hline 2 & 5.2106 & 7.8612 & 9.9654 & 9.4035 & 12.0541 \\
\hline 3 & 6.5648 & 8.5153 & 8.6261 & 9.7565 & 10.8561 \\
\hline \multicolumn{5}{c}{ Table 4 System Reliability Indices } \\
\hline \multirow{2}{*}{ Case } & \multicolumn{5}{c}{ System Reliability Indices } \\
\cline { 2 - 6 } & SAIFI & SAIDI & CAIDI & ASAI & ENS \\
\hline 1 & 1.7312 & 7.9166 & 4.2524 & 99.9159 & 52.9625 \\
\hline 2 & 1.7126 & 6.8541 & 3.6921 & 99.9368 & 38.3039 \\
\hline 3 & 1.7562 & 8.5156 & 5.3562 & 99.8981 & 62.1355 \\
\hline
\end{tabular}

\section{Summary}

Under the $\mathrm{V} 2 \mathrm{G}$ mode, the random behavior of electric vehicle is simulated by the daily travel data and the existing level of the user parameters, the user's random behavior is modeled in time and space. Reliability assessment algorithm is based on the dual sampling, the model established by sampling simulation, then reliability indices of partial load point and system reliability indicators is given in the end, and all the result is proved that the randomness of charge and discharge of electric vehicles have a significant influence on the reliability level of the grid. Some of the parameters of electric vehicles are assumed appropriately in this paper, the impact on distribution network reliability levels of users' random behavior, as well as some practical significance is given by some incentive policies and control strategies of the grid.

\section{References}

[1] LIU Wen-xia, ZHANG Min, Reliability Modeling and Quantitative Analysis of Distribution Network Considering Electric Vehicle Charging and Discharging[J] . Proceedings of the CSU-EPSA. 2013,25(4):1-6

[2] He Jian, Cheng Lin. Reliability Assessment of Composite Power System With Large-Scale Grid-Integration of Plug-in Hybrid Electric Vehicles [J]. Power System Technology 2013,37(4):899-905 
[3] Wu Luwei, Wang Hewu.Beijing-based private passenger travel features GPS research [D]. Beijing: Tsinghua University, 2013.

[4] Taylor M J, Alexander A. Evaluation of the impact of plug-in electric vehicle loading on distribution system operations[C]//EEE Power \& Energy Society General Meeting. Calgary, Canada: IEEE, 2009: 1-6.

[5] Zhang Hui. Reliability Evaluation of Transmission Grid Considering the Uncertainty and Software Development [D]. Beijing: China Electric Power University, 2013.

[6] Chen Can, Wu Wenchuan, Zhang Boming. Reliability evaluation for large-scale distribution network based on component groups[J]. Power System Technology, 2012，36(8): 81-86 